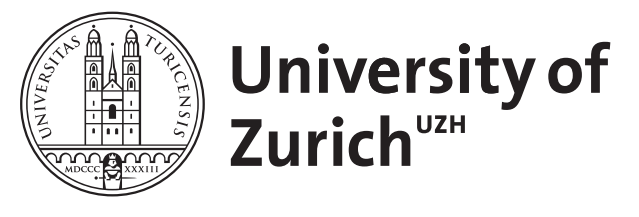

\title{
Sprunggelenkschäden bei Milchkühen - eine Übersicht
}

\author{
Nuss, Karl ; Weidmann, E
}

\begin{abstract}
Chronisch-traumatische Schädigungen des Sprunggelenks gehören zu den häufigsten Technopathien an den Gliedmaßen beim Rind. Die Prävalenzen liegen heutzutage in vielen Betrieben zwischen 40 - 70 \%, was im Hinblick auf das Wohlergehen der Kühe bedenklich ist. Diese Schädigungen sind vor allem Indikatoren für die unzureichende Tiergerechtheit der Liegebo-xen und der Liegeflächen. Haarverlust und Dekubitalstellen an Sprunggelenken sind zudem mit Lahmheiten und weiteren Gliedmassenkrankheiten, aber auch Eutererkrankungen und verringerter Milchleistung assoziiert. Deswegen haben sie nicht nur für das Wohlbefinden, sondern auch für die Leistung von Milchkühen eine Bedeutung. Epidemiologische Studien und Verhaltensuntersuchungen zeigen, dass - korrekte Abmessungen der Liegeboxen voraus-gesetzt - unter den verschiedenen Liegeflächen die Strohmist-Matratze sowie Sand hinsicht-lich der Prävalenz von Liegeschäden am besten abschneiden.
\end{abstract}

DOI: https://doi.org/10.1055/s-0038-1623177

Other titles: Hock lesions in dairy cows - an overview

Posted at the Zurich Open Repository and Archive, University of Zurich

ZORA URL: https://doi.org/10.5167/uzh-89147

Journal Article

Accepted Version

Originally published at:

Nuss, Karl; Weidmann, E (2013). Sprunggelenkschäden bei Milchkühen - eine Übersicht. Tierärztliche Praxis. Ausgabe G, Grosstiere/Nutztiere, 41(G):234-244.

DOI: https://doi.org/10.1055/s-0038-1623177 


\title{
Sprunggelenkschäden bei Milchkühen - eine Übersicht
}

\author{
Karl Nuss, Eva Weidmann \\ Departement für Nutztiere, Vetsuisse-Fakultät der Universität Zürich
}

\section{Zusammenfassung}

Chronisch-traumatische Schädigungen des Sprunggelenks gehören zu den häufigsten Technopathien an den Gliedmaßen beim Rind. Die Prävalenzen liegen heutzutage in vielen Betrieben zwischen $40-70 \%$, was im Hinblick auf das Wohlergehen der Kühe bedenklich ist. Diese Schädigungen sind vor allem Indikatoren für die unzureichende Tiergerechtheit der Liegeboxen und der Liegeflächen. Haarverlust und Dekubitalstellen an Sprunggelenken sind zudem mit Lahmheiten und weiteren Gliedmassenkrankheiten, aber auch Eutererkrankungen und verringerter Milchleistung assoziiert. Deswegen haben sie nicht nur für das Wohlbefinden, sondern auch für die Leistung von Milchkühen eine Bedeutung. Epidemiologische Studien und Verhaltensuntersuchungen zeigen, dass - korrekte Abmessungen der Liegeboxen vorausgesetzt - unter den verschiedenen Liegeflächen die Strohmist-Matratze sowie Sand hinsichtlich der Prävalenz von Liegeschäden am besten abschneiden.

\section{Rind, Lahmheiten, Sprunggelenk, Haarverlust, Dekubitus, Umfangsvermehrung}

\section{Summary: Hock lesions in dairy cows - an overview}

Hock lesions are the most common husbandry-related disease of the locomotor system in dairy cattle. These conditions are referred to as technopathies. The prevalence of tarsal lesions ranges from 40 to $70 \%$ and thus, from a welfare standpoint, clearly exceeds an acceptable level. These lesions usually indicate inadequate stall or cubicle design in dairy barns. The presence and severity of hock lesions are associated with lameness and other limb disorders as well as mastitis and reduced milk yield. Hock lesions therefore influence animal welfare and production. Epidemiological and behavioural studies have shown that a manure pack covered with straw is associated with the lowest prevalence of lesions, followed by sand bedding, provided that the lying space is adequate.

Key words: Cattle, lameness, hock, hair loss, decubitus ulcer, swelling 


\section{Einleitung}

Die hohe Prävalenz von Lahmheiten in Milchkuhbeständen ist besorgniserregend (37, 59). Erkrankungen des Bewegungsapparates nahmen postmortalen Untersuchungen zufolge den größten Anteil (45 \%) an den Ursachen eines spontanen Todes von Milchkühen ein; zudem wiesen $84 \%$ dieser Milchkühe mehr als eine Erkrankung des Bewegungsapparates auf (59, 82). Die Lahmheitsprävalenz wurde als wichtigster Faktor eingestuft, wenn es darum ging, das Wohlergehen von Milchkühen zu beurteilen (1, 54, 88). Fruchtbarkeitsstörungen, Stoffwechselerkrankungen und Verhaltensabweichungen wurden diesbezüglich weniger stark gewichtet $(34,60)$.

Die starke Zunahme der Lahmheitsprävalenzen geht auch mit der Einführung und Verbreitung der Liegeboxen-Laufstall-Haltung und mit der Zunahme der Bestandgrößen einher. Im Jahr 2004 wurden mehr als zwei Drittel aller Kühe in Deutschland in Laufställen gehalten, gebietsweise waren es 96 \% (42). In der Anbindehaltung lagen die Lahmheitsprävalenzen infolge der geringeren Beanspruchung der Klauen in der Regel tiefer als in Laufställen $(6,17,96)$. Kühe, die im Anbindestall mit Stroheinstreu oder in Systemen mit eingestreuten Liegeflächen gehalten wurden, wiesen eine längere Nutzungsdauer auf als Kühe aus Boxensystemen (9). Bei der ganzjährigen Stallhaltung wirkt sich das Fehlen einer Erholungsphase für die Klauen und Gliedmaßen während des Weidegangs (33) ungünstig aus.

Sprunggelenkschädigungen werden zu den „Technopathien“ gerechnet. Dieser Begriff bezeichnet beim Rind eine Schädigung oder eine Erkrankung, die durch schlechte Stalleinrichtungen, insbesondere raue Liege- oder Laufflächen oder falsche Abmessungen der Liegeboxen, hervorgerufen wird (21, 80, 95). Zu diesen Schädigungen zählen im engeren Sinn Haarverlust, Hautschädigung, Dekubitalnekrosen und Umfangsvermehrungen. Aber auch Klauenerkrankungen, die durch vermehrten Abrieb des Sohlenhorns infolge rauer oder abrasiver Stallböden bedingt sind, und bestimmte Gliedmaßenerkrankungen wie Osteochondrose und Entzündung der Wachstumszonen, die auf harten Böden vermehrt vorkommen (79), fallen unter diesen Begriff. Gehäuft auftretende akute Verletzungen an scharfkantigen Metalleinrichtungen oder dem Mistschieber sind ebenfalls dazu zu rechnen.

Allerdings sind Sprunggelenkschäden nicht nur in Laufställen, sondern auch in vielen Anbindeställen häufig, selbst wenn Weidegang möglich ist (10, 93). Zu dem Zeitpunkt, zu dem viele der heutigen Ställe gebaut und eingerichtet wurden, bestimmten vorwiegend arbeitswirt- 
schaftliche Gesichtspunkte die Details der Planung und Ausführung (35, 48, 56, 76). Mit der Abnutzung der Liegeflächen und der zunehmenden Körpergröße der Milchkühe treten jedoch vermehrt Schäden auf (96). In Deutschland muss Tieren laut $§ 2$ Abs. 1 TierSchG, 2010 eine verhaltensgerechte Unterbringung geschaffen werden; dabei darf die Möglichkeit zu artgemäBer Bewegung nicht so eingeschränkt werden, dass ihnen Schmerzen oder vermeidbare Leiden oder Schäden zugefügt werden.

Die Schmerzen oder Leiden, die durch Haarverlust, Hautschädigung oder Umfangsvermehrungen am Sprunggelenk entstehen, sind nicht genau quantifizierbar. Tierärzte schätzten die damit verbundenen Schmerzen auf einer zehn Punkte umfassenden Skala mit den Graden drei bis fünf (Median: 5) ein (38).

Aus diesen Gründen wurden in den letzten Jahren viele Untersuchungen durchgeführt, mit den Zielen, die Bedürfnisse der Milchkühe zu erforschen (31, 32, 53, 76), eine bessere Haltungsumwelt für sie zu schaffen und damit ihre Leistungsfähigkeit zu erhalten. Gängige Schlagworte dieser Forschungsrichtung sind „Kuhkomfort“, „Tiergerechtheit“ und „kuhbasierte“ Untersuchungen $(22,40,57,74,81,86)$. Die vorliegende Übersicht beschäftigt sich vorwiegend mit den Technopathien des Sprunggelenks beim Rind.

\section{Lokalisationen und Arten von haltungsbedingten Schäden}

Infolge wiederholter Traumata entstehende Schädigungen können bei Milchkühen grundsätzlich an allen Körperstellen auftreten, die regelmäßiger Überlastung, beispielsweise durch Druck oder Reibung, ausgesetzt sind $(44,67)$. Obwohl die Sprunggelenke am häufigsten betroffen sind, kommen gleichartige Veränderungen an Karpus und Knie, aber auch am Oberschenkel, Ellbogen oder an Nacken und Widerrist vor. Diese Schädigungen können gleichermaßen wie im Folgenden für die Sprunggelenkschäden dargestellt klassifiziert werden (71).

Drei Schadensarten werden im Allgemeinen unterschieden: Haarverluste, Dekubitus und Umfangsvermehrungen. Sie entstehen, wenn Kühe in den Boxengassen, vor dem Melkbereich oder beim Aufsteh- oder Abliegevorgang mit den Boxenabtrennungen kollidieren (55), oder wenn sie auf einer ungepolsterten, harten Liegefläche liegen müssen (13, 28, 45, 71, 76, 84, $87,96,97)$. 
Beim erschwerten Aufsteh- oder Abliegevorgang treten Haut und Gummimatten/Betonböden in Reibung (55). Durch die begleitende Wärmeentwicklung und bei bestehender Feuchtigkeit werden die Haare leichter abgerieben. Naheliegend ist, dass infolgedessen eine isolierende Polsterschicht fehlt, die die Haut vor mechanischen Schäden schützt. Zudem können chemische Bestandteile von Exkrementen die Haut oder darunter liegendes Gewebe (62) mazerieren. Fehlendes Haarkleid und Hautschädigung stellen die erste Stufe von Dekubitalerkrankungen dar. Dekubitalschäden entstehen besonders über schlecht gepolsterten Knochenregionen (Abb. 1 a) durch den Druck des Körpergewichts, wenn ein unnachgiebiger Liegebereich Durchblutungsstörungen in der Haut und den darunter liegenden Weichteilen induziert. Kühe unternehmen in zu eng bemessenen Boxen oft mehrere Aufstehversuche (29), wobei die Haut der Sprung- und Karpalgelenke ebenfalls vermehrt abgerieben und geschädigt wird. Es ist anzunehmen, dass bei jedem Kontakt der Gelenke mit der harten Oberfläche Schmerzen auftreten (42). Dieser Kontakt dauert umso länger, je weniger tiergerecht die Liegefläche ist, weil die Tiere, vermutlich aus Angst vor Schäden und Schmerzen, längere Zeit für den Abliegeund Aufstehvorgang brauchen $(48,53)$.

Die dritte Schadensart, die Umfangsvermehrung, entsteht im einfachsten Fall durch eine Verdickung der Haut (,Schwielenbildung“). Bei Überbeanspruchung der Haut und Unterhaut treten jedoch bald eine Lockerung der Subkutis und des Bindegewebes mit Serombildung in den Gewebsspalten hinzu (Abb. 1 b). Aufgrund mikrobieller Invasion der geschädigten Haut und Infektion der Unterhaut entstehen leicht akute entzündliche Ödeme und Phlegmonen. Die Überbeanspruchung kann, wenn keine Infektion stattfindet, kompensatorisch zu der Bildung eines Hygroms führen. Dieses Hygrom kann die Belastung kompensieren, es kann aber auch wachsen (Abb. 1 a) und sich nach bakterieller Besiedelung zu einem Abszess entwickeln.

Hinsichtlich der Pathogenese der einzelnen Läsionen kann nicht von einer linearen Entwicklung, beginnend mit Haarverlust, fortschreitend mit Dekubitus und letztlich resultierend in Umfangsvermehrungen (27) ausgegangen werden. Die Schädigungen treten gemeinsam oder isoliert auf, jedoch wurden jeweils unterschiedliche Risikofaktoren für sie identifiziert (15, 71). Jede der drei Läsionen hat vermutlich einen unterschiedlichen Einfluss auf das Wohlergehen von Rindern (52).

Die unzureichende Definition des Ausdrucks „Sprunggelenkläsion“ wurde durch die Einführung eines Klassifizierungssystems mit Hilfe einer „Sprunggelenk-Karte“ (Abb. 2) verbessert 
$(52,71)$. Die Beurteilung der Schwere der Läsionen bleibt zwar weiterhin subjektiv, aber die Prävalenz der verschiedenen Läsionen kann zuverlässiger bestimmt werden. Jede Art der Schädigung wird in vier Schweregrade $(0=$ nicht vorhanden, $1=$ leicht, $2=$ mäßig und $3=$ schwer) unterteilt. Die Fläche des Haarverlusts oder des Dekubitus und die Größe der Umfangsvermehrung bestimmen die Schweregrade (Abb. 3).

Als Folge von Sprunggelenkschädigungen können eitrig-abszedierende Phlegmonen, Sehnenscheiden-, Schleimbeutel- oder Gelenkentzündungen auftreten (36). In solch fortgeschrittenen Fällen sind oftmals auch Muskeln und Gelenkbänder sowie Knochen infiziert und nekrotisch verändert $(47,67)$. Diese schweren Formen der Schäden sind mit Abmagerung, starken Schmerzen und oftmals Festliegen verbunden.

\section{Prävalenz}

Bei Milchkühen, die auf Tiefstreu oder ganzjährig auf der Weide gehalten werden, sind Technopathien nahezu unbekannt $(30,33,36,43,50,78)$. Die meisten Untersuchungen über Technopathien liegen im Zusammenhang mit Liegeboxenlaufställen (3, 27, 46, 55, 65, 72, 89), weniger mit Anbindeställen (73, 93) vor.

Sprunggelenkschädigungen bei Rindern sind in Großbritannien (76, 91), Kontinentaleuropa $(43,45,46)$ und Nordamerika von allen Technopathien am besten dokumentiert $(27,45,56$, 89). Bisher vorliegende Untersuchungen verwendeten jedoch kein einheitliches Beurteilungssystem, beurteilten nur eine Gliedmaße oder nur schwerwiegende Schäden $(71,72)$, so dass die Angaben über Prävalenzen (Tab. 1) auf unterschiedlichen Beurteilungen beruhen. Die hohe Prävalenz von Sprunggelenkläsionen $(45,71,72,90,97)$ wird im Hinblick auf das Wohlergehen der Kühe als bedenklich oder inakzeptabel eingestuft $(76,87)$. Liegeschäden und Lahmheiten haben gemeinsame hohe Prävalenzen $(45,61)$, auch weil lahme Kühe für längere Zeit liegen als nicht lahme $(8,14)$.

Aber auch Karpalgelenkveränderungen wiesen hohe Prävalenzen von $35.3 \pm \underline{25.7 \%}$ (45), 31,5 $\%$ (53) und 24-43\% (93) auf. Weitere Technopathien, wie die Entzündung der Karpalgelenkstrecker (47) oder die Nekrose des lateralen Kollateralbands am Knie (36, 67), wurden zwar eingehend klinisch und pathologisch, aber bisher nicht auf ihre Prävalenz auf Bestandesebene hin untersucht. Eine detaillierte Untersuchung auf Bestandesebene wäre aber notwen- 
dig, da insbesondere leichte Schädigungen nicht als Erkrankung wahrgenommen, sondern erst

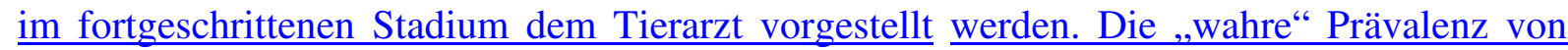
$\underline{\text { Schäden kann daher nur erfasst werden, wenn auch leichte Veränderungen systematisch und }}$ detailliert registriert werden (93).

Von 6‘896 Sprunggelenken, die von Potterton et al. (2011) mit der obengenannten „Sprunggelenk-Karte“ klassifiziert wurden, wiesen 81,4 \% haarlose Stellen, 16,5 \% Dekubitalschäden und 22,1\% deutliche Umfangsvermehrungen auf (Tab. 1) (72). Die Läsionen waren oft, zu 80-85\%, an beiden Hintergliedmaßen gleichzeitig zu finden $(72,89)$, in einer anderen Untersuchung (93) aber nur zu $40 \%$. Obwohl die Schäden meist geringgradig waren, traten schwere Dekubitalschäden immerhin mit einem Anteil von $2.1 \%$ und starke Umfangsvermehrungen mit einem Anteil von 2,0 \% auf (72). In anderen Untersuchungen $(57,89)$ erreichten schwere Schäden einen Anteil von 0.5 bis $4.1 \%$. In einzelnen Problembetrieben waren sogar $20 \%$ der Kühe von schweren Dekubitalerkrankungen oder Umfangsvermehrungen betroffen (72). Die

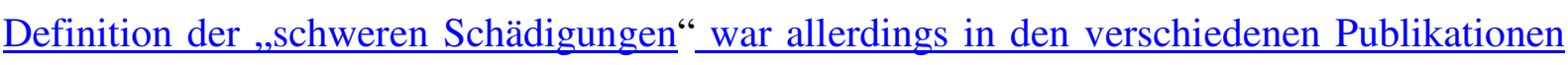
nicht einheitlich.

Nur wenige Autoren $(48,55,56,92)$ führten die Untersuchungen über einen längeren Zeitraum durch und untersuchten die Entwicklung der Sprunggelenkläsionen. In diesen war jedoch offensichtlich, dass sich die auf Läsionen auf harten Liegeflächen verschlimmerten, während sie sich auf weicher, verformbarer Einstreu besserten $(48,55)$.

\section{Risikofaktoren}

\section{Liegezeiten}

Milchkühe streben danach, etwa 50 bis $60 \%$ ihrer täglichen Zeit mit Liegen zu verbringen $(41,42,63)$. Sie liegen somit üblicherweise 12 bis 14 Stunden und in neun bis elf Liegeperioden zu 80 bis 90 Minuten (41). Sie ziehen das Liegen unter experimentellen Voraussetzungen sogar Fressen und Sozialkontakten vor (63). Ruhe- und Liegezeiten sind wichtig für die Erholung und den Erhalt der Leistung (49, 70). Das (gestörte) Liegeverhalten und die Liegezeit eignen sich daher besonders als Indikatoren für tiergerechte Haltung und Wohlbefinden (29, 39, 93). Sie werden durch viele Faktoren beeinflusst (Tab. 2). In einem Anbindestall mit Be- 
tonliegeflächen verlängerten sich paradoxerweise die Liegezeiten, vermutlich aber deshalb, weil das schmerzhafte Aufstehen und Abliegen vermieden wurde $(29,48)$.

Milchkühe verbrachten, wenn sie die freie Wahl hatten, signifikant $(\mathrm{P}<0.001)$ mehr Zeit auf Sand und Stroh (44.1\% und 33,2 \% der gesamten Liegezeit) als auf Gummimatten oder Gummimatratzen (11.6\% oder 11.1\%) (11). Weiterhin war die Dauer einer Liegephase_auf

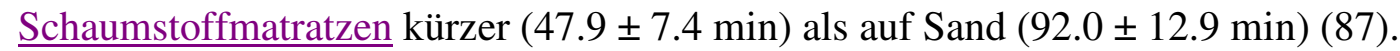

Dabei zeigte es sich, dass ein Mehr an Einstreu auch die Liegezeiten deutlich erhöhte (86). Für jeden Zentimeter fehlenden Polstermaterials reduzierte sich die tägliche Liegezeit um 11 Minuten. Für 13,7 Zentimeter fehlendes Polstermaterial wurden 2,33 Stunden weniger Liegezeit, verglichen mit einer_gut gepflegten Sandliegebox, festgestellt (19).

Boxen mit trockenem Polstermaterial oder trockener Liegefläche wurden erheblich besser genutzt als feuchte (74). Die Liegezeit verlängerte sich um etwa 5 Stunden pro Tag, wenn trockene anstatt feuchte Liegeflächen zur Verfügung standen (25). Wenn Kühe die Wahl zwischen trockenen und feuchten Liegeflächen hatten, lagen sie fast ausschließlich auf den trockenen (Tab. 2).

\section{Polstermaterialien}

Liegeboxen-Laufställe können nach der Gestaltung der Liegeboxen und der Menge des Polstermaterials in verschiedene Kategorien unterteilt werden $(48,88)$ : Hochbox, Tiefbox und Hoch-Tiefbox. Die Liegefläche in einer Hochbox ist gegenüber dem Niveau der Lauffläche erhöht und üblicherweise mit einer Gummimatte oder Gummimatratze belegt. Die Grundfläche der Tiefbox liegt hingegen auf gleicher Ebene wie die Lauffläche des Stalles. Durch die Einstreuschicht wird die Liegefläche dann ebenfalls_angehoben. Die Tiefbox mit StrohMistmatratze weist die größte Grundfläche aller Liegeboxentypen auf (88), auch weil die Streuschwelle, die Begrenzung zum Laufgang hin, nicht zur Liegefläche gerechnet werden darf (20). Die Hoch-Tiefboxe kombiniert das Merkmal der Hochboxe mit über dem Laufgang gelegener Grundfläche mit der Streuschwelle und Einstreu der Tiefboxe.

Generell bevorzugen Milchkühe weiche, trockene Liegeflächen (57, 86, 90). Sie ziehen stroheingestreute Liegeboxen meist sogar Sandboxen, denen sonst die besten Eigenschaften hinsichtlich Gliedmassengesundheit zugeschrieben werden, vor (65). Die Tiefe der Einstreu ist 
für die Kühe insofern von Bedeutung, dass die besser eingestreuten Boxen den weniger tief gepolsterten Boxen vorgezogen werden (19). Die Polster- und Einstreuschicht sollte etwa 25 $\mathrm{cm}$ tief sein. Die Einstreuschicht muss immer wieder aufbereitet werden, damit sich keine Mulden bilden, bei denen an der tiefsten Stelle der harte Boden zum Vorschein kommt (Tab. 2).

Viele unterschiedliche Liegeflächen und Polstermaterialien - Langstreu, Kurzstreu, Sägemehl, Sägespäne, Sand, geotextile Matten, Gummimatten, gepolsterte Gummimatten, wassergefüllte Matten - wurden hinsichtlich der Akzeptanz durch Kühe untersucht (25, 36, 66, 71, 75, 83, 87). Das Polstermaterial der Liegefläche war ein gemeinsamer Risikofaktor für das Entstehen von Haarverlust und Umfangsvermehrung am Tarsus (71). Bei Stroheinstreu war die Prävalenz von Haarverlust und Umfangsvermehrungen im Vergleich zu Sägespänen geringer (76); allerdings traf das nur für Lang-, nicht für Kurzstreu zu. Die gesamte Liegefläche musste dabei mit Einstreu abgedeckt sein. Grundsätzlich waren Haarverlust und Dekubital-

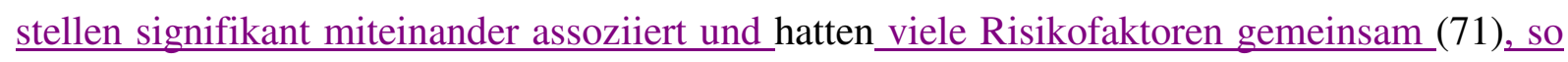
dass angenommen werden kann, dass die Entstehungsweise ähnlich ist. Umfangsvermehrungen hingegen scheinen andere Risikofaktoren zu haben (71).

Solide Gummimatten, aber auch gepolsterte Gummimatten stellten Risikofaktoren für die Entwicklung von Sprunggelenkläsionen dar (3, 55, 71, 87); mit einem höheren Alter der Gummimatten traten Haarverluste vermehrt auf. Die Gefahr von Sprunggelenkschäden nahm bei der Haltung auf Hochboxen mit Gummimatten bzw. -matratzen im Vergleich zu Tiefboxen mit Stroh-Mist-Matratze stark zu (42). Obwohl gepolsterte Matratzen weniger Schäden als Gummimatten verursachten, waren die Läsionen doch häufiger und stärker als bei Tiefstreuhaltung $(15,18,55,89)$ Die Schäden am Sprunggelenkwaren bei Sägemehleinstreu grösser, wenn die Liegeplätze kürzer waren (89). Sägemehl verstärkte auch die Reibung von Gummimatten, sodass diese Kombination regelrecht abrasiv wirkte (36). Etwas gegensätzliche Ergebnisse hinsichtlich Sprunggelenkläsionen erbrachte der Vergleich von Stroh- und Sandeinstreu (65), weil in dieser Untersuchung die Sandbox besser abschnitt als die Strohbox. Nur in einer Untersuchung (58) wurde festgestellt, dass Kühe Sandboxen mieden.

In einer Untersuchung, die Hochboxen mit geotextilen Matten und Sägemehleinstreu einerseits und Tiefboxen mit Sand als Unterlage andererseits verglich, entstanden bei den meisten Kühen auf Hochboxen innerhalb der ersten 6 Wochen der Laktation Schäden an der lateralen 
Fläche des Tarsus $(89,90)$. Die Sprunggelenkschäden entwickelten sich in Betrieben mit den geotextilen Matratzen weitaus schneller und die Veränderungen waren schwerer. Bei Sandboxen (Tiefboxen) waren lediglich Schädigungen am proximalen Ende des Tuber calcanei häufiger, vermutlich weil diese Stelle an der Streuschwelle aufgerieben wurde, was bei der ebenen Liegefläche der Hochboxe nicht möglich war $(45,90)$.

\section{Abliege- und Aufstehverhalten}

Es wurde geschätzt, dass $26 \%$ der in Boxenlaufställen gehaltenen Kühen Aufstehschwierigkeiten zeigen (36). Dementsprechend wurden in den Hochboxen viele Ablege- und Aufstehversuche, eine kürzere Liegedauer und weniger Komfortverhalten beobachtet (88), dafür traten vermehrt Technopathien_auf. Die verlängerte Dauer des Abliegevorgangs war mit Tarsalund Karpalgelenkschäden assoziiert (61). Die Zeit, in der Kühe vor dem Abliegen in der Boxe standen, betrug bei Stroheinstreu 5,2 Minuten, bei Gummimatten aber 17,2 Minuten (48). Auch zum Abliegen selbst benötigten Kühe erheblich mehr Zeit auf Schaumstoffmatratzen als auf Sand oder Stroh-Mistmatratzen. In wenig gepolsterten Hochboxen wurden 20,4\% der Abliegevorgänge abgebrochen, in eingestreuten Hochtiefboxen dagegen nur $4 \%$ (48). Mehrfache und längere Zeit andauernde Aufstehversuche (35) und pferdeartiges Aufstehen traumatisieren Karpal- und Sprunggelenke (51).

Weiterhin veränderten Lahmheiten die Art des Aufstehens dahingehend, dass mehr Versuche benötigt wurden, bis die Tiere standen $(48,71)$. Wenn man annimmt, dass Kühe auf der Weide für das Abliegen bis zu drei Meter Platz in Längsrichtung benötigen und im Liegen der seitliche Platzbedarf 135 und mehr cm beträgt (20), sind auch großzügigere Richtwerte für die Boxenabmessungen (Tab. 3) zu klein.

\section{Abmessungen der Liegeboxen}

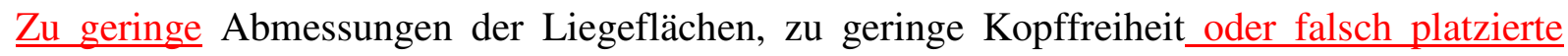
Nackenrohre können bewirken, dass die Kühe im Abliege- und Aufstehverhalten stark eingeschränkt sind. Insbesondere hochträchtige Färsen und Kühe haben Probleme, mit ungeeigneten Boxen oder Anbindeplätzen zurecht zu kommen (47, 67). Längere Liegeplätze, insbesondere mit Stroheinstreu, waren mit weniger Schäden verbunden (43). Allerdings nahmen bei zu 
langen Liegeboxen (> $260 \mathrm{~cm}$ ) die Schädigungen wieder zu (45), ohne dass eine Erklärung

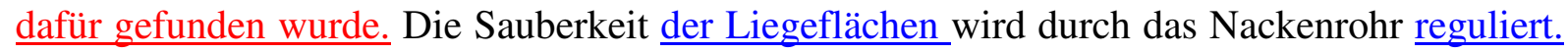
Die Einstellung des Nackenrohrs ist oft zu knapp, weil die Präferenzen auf die Vermeidung der Verschmutzung der Liegefläche durch Kot und Harn gelegt werden, auch um Mastitiden vorzubeugen und Arbeitsstunden zu reduzieren $(7,26)$. Zugleich geht dies aber zu Lasten der Liegebequemlichkeit und des ungestörten Aufsteh- und Abliegevorgangs; Lahmheiten und Sprunggelenkschäden nehmen zu (93). Das Zurücksetzen des Nackenrohrs um wenige Zentimeter kann auch ausreichend lange Boxen ungeeignet machen $(44,45,85)$. Wenn das Nackenrohr von 114 Zentimeter auf 127 Zentimeter angehoben wurde, verlängerte sich die Liegezeit von Kühen um 2,1 Stunden pro Kuh und Tag (64). Diese positiven Effekte zeigten sich auch hinsichtlich des Haarverlusts an Sprunggelenken: Wenn das Nackenrohr höher als 115 cm vom Boxenboden und weiter als $199 \mathrm{~cm}$ vom Boxenende entfernt war, war das Risiko des Haarverlust signifikant niedriger als bei geringeren Abmessungen (71). Sowohl für die Höhe des Nackenrohrs als auch für dessen Entfernung von der der Liegekante gibt es unterschiedliche Angaben (Tab. 3). Eine Nackenrohrhöhe von $110 \mathrm{~cm}$ wird als zu gering angesehen. Für Deutsche Holstein-Friesian-Kühe wird eine Höhe zwischen 125 und $133 \mathrm{~cm}$ empfohlen, wobei die horizontale Entfernung von der Kotstufe bzw. Streuschwelle 165-175 cm betragen soll (68).

\section{Sonstige Faktoren}

Sprunggelenkschäden, insbesondere Haarverlust und Dekubitalstellen, waren mit Lahmheiten und Klauenkrankheiten $(3,5,6,27,30,45)$ mit Liegeschäden am Karpus (93), aber auch Eutererkrankungen und verringerter Milchleistung $(4,27,48,77)$ assoziiert.

Die Anzahl der Sprunggelenkschäden erhöhte sich auch mit steigendem Gefälle der Liegeflächen (30). Bei starkem Gefälle rutschten die Kühe nach hinten und die Sprunggelenke kamen über der Kante der Liegefläche zu liegen (Abb. 4). Im Allgemeinen gilt es zudem zu bemerken, dass die Nutzung von Liegeflächen nicht nur von ihren Eigenschaften (Trockenheit, Weichheit), sondern auch von der Gewöhnung der Rinder abhängig_war (7, 13, 65, 86).

Weitere, weniger spezifische Risikofaktoren für Sprunggelenkschäden sind Milchleistung, Trächtigkeitsstadium (45), Rasse und Herdengröße (71), Body condition score und Alter. Sprunggelenkschäden sind bei Laktationsbeginn besonders häufig, was auf Aufstehschwierig- 
keiten im Abkalbezeitraum, aber auch die Besonderheiten der sozialen Integration nach Umgruppierung von Kühen hinweist (45, 93). Eine hohe Besatzdichte im Bestand hat einen negativen Einfluss auf die Liegezeiten (81). Die Zeit pro Jahr, in der sich die Kühe im Stall aufhalten, wirkt sich ebenfalls negativ auf die Sprunggelenkintegrität aus (71). Die Sprunggelenkschäden sind nach einigen Untersuchungen bei einem niedrigen Body condition score (BCS) häufiger festzustellen $(18,45)$, wohingegen bei Anbindeställen die Kühe mit höherem BCS eher Schäden zeigten (73). Die Schweregrade der Schäden nehmen mit dem Alter zu (45, 71, 76, 89); allerdings wiesen Erstkalbende in Anbindeställen nach einer Untersuchung (2) das höchste Risiko für Sprunggelenkschäden auf.

Weiterhin waren chemische Produkte (Kalk, Formaldehyd), die auf die Liegefläche aufgebracht wurden, mit dem Auftreten von Haarverlust und Umfangsvermehrungen assoziiert. Diese Substanzen irritieren, zusammen mit der Feuchtigkeit auf den Matten, die Haut und verursachen bei nicht fachgerechter Anwendung Verätzungen (71).

\section{Therapiemaßnahmen und Prävention}

Therapiemaßnahmen sind üblicherweise Einzeltieren mit akuten entzündlichen Gewebeveränderungen (Abb. 5 a und b), die auffallende klinische Symptome nach sich ziehen, vorbehalten. Akute Phlegmonen werden mit Verbänden und Antibiotika nach den-geltenden Richtlinien sowie nicht zuletzt durch Aufstallung in einer gut eingestreuten, trockenen Krankenboxe behandelt. Infizierte Hygrome werden gegebenenfalls inzidiert und drainiert, wonach gleichfalls eine zeitlich begrenzte gesonderte Aufstellung auf Stroh notwendig ist. Bei Zuchttieren können gut abgegrenzte Hygrome (Abb. 5 b) auch in toto exzidiert werden (69). All diese Behandlungen ziehen eine aufwendige Betreuung und Nachsorge nach sich und verlangen eine gesonderte Aufstallung auf weicher, trockener Einstreu (Tiefstreuareal). Einzeltiere mit leichten Schäden, wie sie Haarverlust, Dekubitus oder Schwellungen meist darstellen, werden $\underline{\text { dem Tierarzt meist nicht zur Behandlung vorgestellt. }}$

Chronische, eitrig-abszedierende Phlegmonen oder bis auf die Kollateralbänder reichende Dekubitalschäden (Abb. 5 a) haben eine ungünstige Prognose $(62,67)$, die Rinder sollten geschlachtet werden. 
Die auf Herdenebene relevanten Maßnahmen zielen auf die Prävention der Sprunggelenkveränderungen, sprich auf die Optimierung von Boxengröße und der Polsterung der Liegeflächen ab. Angaben zu Liegeboxenabmessungen stellen einen Kompromiss dar (20). Milchkühen sollten generell besonders große und bequeme Ruhebereiche zur Verfügung gestellt werden (16). Die Abmessungen (Tab. 3) von Liegeboxen sollten sich prinzipiell nach den größeren Kühen eines Bestandes richten. Für die ideale Liegefläche müssen jedoch nicht nur die Tier-

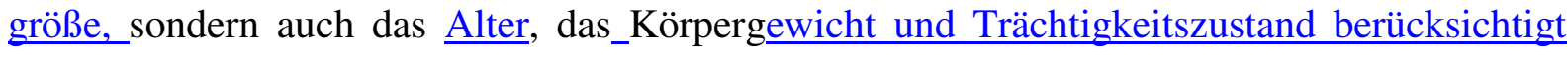
werden. Der individuelle Bedarf einer Kuh kann nach der Formel Liegelänge $=1,11$ x schräge Rumpflänge $+20 \mathrm{~cm}$ Sicherheitszuschlag berechnet werden (41). Der Kopfraum sollte dabei mindestens $60-80 \mathrm{~cm}$ betragen. Die lichte Breite der Liegeboxe wird nach der Formel Widerristhöhe $x$ 0,85 abgeschätzt. Die Position des Nackenrohrs kann nach der Formel Rumpflänge (Schwanzansatz-Widerrist) + $5 \mathrm{~cm}$ (68) bestimmt werden.

Nur wenige Maßnahmen, die eine Verbesserung erbringen, können sofort ergriffen werden. So wurden beispielsweise zur Verbesserung der Liegefläche von Hochboxen diese in sogenannte Hochtiefboxen umgebaut. Hierzu wurden ein Holzbalken an die hintere Boxenkante sowie eine Bugschwelle angebracht, so dass die Fläche tief eingestreut werden konnte (48). Durch diese Maßnahmen verringerten sich die Sprunggelenkschäden signifikant. Eine weitere einfach durchzuführende, erfolgversprechende Maßnahme ist die Versetzung des metallenen Nackenriegels oder sein Ersatz durch flexiblere Materialien $(7,64)$.

Eine Überbelegung des Stalls ist unbedingt zu vermeiden. Mindestens eine Box pro Kuh sollte vorhanden sein. Ein größeres Flächenangebot in den Liegeboxen und freie Liegeflächen üben einen günstigen Einfluss auf die Klauen- und Gliedmassengesundheit aus. Zudem haben das Platzangebot im Laufgang und die Beschaffenheit der Oberflächen einen signifikanten Einfluss auf die Prävalenz von Lahmheiten (88). Klauenkrankheiten müssen behandelt werden, da Technopathien und Lahmheiten sich negativ beeinflussen, indem lahme Tiere mehr liegen und erschwert aufstehen, wodurch die Zeitdauer der Exposition der Gelenke erhöht ist.

Zuletzt hat sich gezeigt, dass der verhaltensbewusste, schonende Umgang mit den Milchkühen und die Einstellung der Tierbesitzer zur Schmerzempfindlichkeit bei Tieren eine Auswirkung auf den Gesundheitszustand und die Produktivität der Milchkühe hat (31, 32, 72). Besitzer, die der Meinung waren, dass Sprunggelenkläsionen schmerzhaft waren, zeigten eine höhere Bereitschaft, etwas dagegen zu unternehmen (72). Viele Betriebsleiter aber registrieren 
Haarverlust und oberflächliche Dekubitalerkrankungen nicht oder betrachten sie nicht als Schäden $(71,72)$, weil sie relativ selten klinischen Konsequenzen nach sich ziehen. Sie empfinden sie vermutlich deswegen als „,normal“, weil sie so häufig sind (52). Verschiedene einfache Tests können zweifelnden Besitzern gezeigt werden, damit sie sich der Qualität der Liegeflächen bewusst werden (36): Der „Kniefalltest“ prüft die Weichheit und Tiefe der Liegefläche (und sollte besser nicht bei ungepolsterten Liegeflächen angewendet werden). Beim „Knöcheltest“ werden die Fingerkuppen oder die Knöchel der Hand auf die Liegefläche aufgesetzt und hin und her gerieben. Die Hitzeentwicklung und die Rauigkeit der Oberfläche und die Abrasivität der Liegefläche werden so abgeschätzt (36). Beim „Liegetest“ legt sich der Untersucher einige Zeit auf die Liegefläche und beurteilt deren Bequemlichkeit und Wärme sowie allfällige Luftbewegungen.

\section{Schlussfolgerungen}

Haarlosigkeit, Dekubitus und Umfangsvermehrung sind schmerzhaft und mit Lahmheiten,

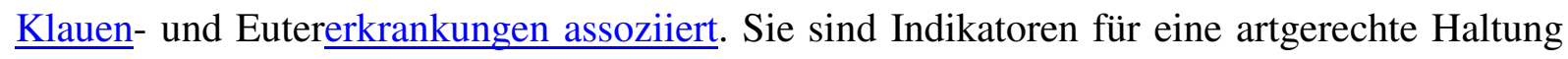
und stellen Vorstufen schwerwiegender Schäden dar. Der wichtigste ursächliche Faktor ist die Liegefläche $(45,55,65)$. Harte und raue Liegeflächen sind mit mehr Schäden assoziiert, wobei blanke Betonflächen schlechter abschneiden als ungepolsterte Gummimatten und diese wiederum schlechter als Gummimatratzen. Kurze Einstreu, Sägespäne, Holzschnitzel und andere sind als Polstermaterialien wenig effektiv und erhöhen teilweise die Abrasivität an den Gelenken. Tiefe, regelmäßig aufbereitete Sand- oder Mistmatratzen-Boxen schneiden am besten ab. Neben der Polsterung sind die Abgrenzungen der Liegeflächen die Schlüsselfaktoren für die Vermeidung der oben genannten Schäden (57). Kühe müssen mit ausreichend großen, gut gepolsterten, trittsicheren, trockenen Liegeflächen, die frei von Schmutz und Verletzungsmöglichkeiten sind, versorgt werden (53). 
Tab. 1 Prävalenz und Arten von Sprunggelenkläsionen

\begin{tabular}{|c|c|c|c|c|}
\hline Autoren & $\begin{array}{l}\text { Prävalenz ins- } \\
\text { gesamt }\end{array}$ & Haarverlust & $\begin{array}{l}\text { Rötung/ } \\
\text { Ulzeration }\end{array}$ & $\begin{array}{l}\text { Umfangs- } \\
\text { vermehrung }\end{array}$ \\
\hline Weary and Tazskun (2000)* & $73 \%$ & k. A. & k. A. & k. A. \\
\hline Klaas et al. (2003) & $63 \%$ & $41.3 \%$ & $4.2 \%$ & $14.2 \%$ \\
\hline Regula et al. (2004) ** & $8-21 \%$ & k. A. & k. A. & k. A. \\
\hline Zurbrigg et al. (2005)*** & $42 \%$ & $36 \%$ & $8 \%$ & $14 \%$ \\
\hline $\begin{array}{l}\text { Kanswohl und Sanftleben } \\
\text { (2006) }\end{array}$ & $\begin{array}{c}55 \% \text { I_25 } \\
\% * * * *\end{array}$ & $46,5 \% / 25 \%$ & $18,3 \% \_7 \%$ & $8,4 \% / 0 \%$ \\
\hline Rutherford et al. (2008) & $49,1 \%$ & 39 & & $8 \%$ \\
\hline Kielland et al. (2009) & $60,5 \% * * * * *$ & $53 \%$ & $6 \%$ & $1 \%$ \\
\hline Liebhart (2009)****** & $49,5 \% / 35,4 \%$ & $22.3 \% / 33.8 \%$ & $27.2 \% / 1.6 \%$ & $14.2 \% / 8 \%$ \\
\hline Lombard et al. (2010) & $23,5 \%$ & k. A. & k. A. & k. A. \\
\hline Potterton et al. (2011) & k. A. & $81,4 \%$ & $16,5 \%$ & $22 \%$ \\
\hline Zaffino (2012) & $\begin{array}{l}56 \% / 47 \% \\
* * * * * * *\end{array}$ & ca. $24 \% /$ ca. $13 \%$ & \multicolumn{2}{|c|}{ ca. $40 \%$ / ca. $34 \%$} \\
\hline
\end{tabular}

k. A. keine expliziten Angaben

* Laufställe, Prävalenz bei geotextilen Matratzen $91 \%$, bei Sand $24 \%$.

** Anbinde- und Laufställe, mit Auslauf/Weidegang

*** Anbindeställe

**** Hochboxe/Tiefboxe

***** Odds ratio (OR) für weiche Liegeflächen 0.22, und für harte Liegeflächen und Gummimatten

0.62

****** Integumentschaden lateral am Tarsus: alter Stall/neuer Stall

******** Anbindestall/Laufstall, Mittelwert 
Tab. 2 Liegezeiten und ihre Abhängigkeit von verschiedenen Faktoren

\begin{tabular}{|c|c|c|}
\hline Faktor & $\begin{array}{l}\text { Auto- } \\
\text { ren }\end{array}$ & Liegezeiten / Liegephasen \\
\hline Menge der Einstreu & (86) & $\begin{array}{l}12 \text { Minuten längere Liegedauer für jedes zusätzliche Kilogramm } \\
\text { Stroh }\end{array}$ \\
\hline Kuhlenbildung & (19) & $\begin{array}{l}\text { Verkürzung der Liegezeit um 2,33 Stunden/Tag bei Verminderung } \\
\text { der Höhe der Liegefläche um mehr als } 13 \mathrm{~cm} \text { unterhalb der Ober- } \\
\text { kante der Liegeflächenbegrenzung }\end{array}$ \\
\hline $\begin{array}{l}\text { Polstermaterial/- } \\
\text { qualität }\end{array}$ & $\begin{array}{l}(15) \\
(11) \\
(48) \\
(83)\end{array}$ & $\begin{array}{l}\text { länger auf Gummimatratzen als auf Gummimatten } \\
\text { Liegeflächen-Wahlversuch: } 44,1 \% \text { der Gesamtliegezeit in Sandbo- } \\
\text { xen, 33,2 \% in Strohboxen, 11,6 \% auf Gummimatten, 11,1\% auf } \\
\text { Gummimatratzen } \\
40 \text { Minuten längere Liegezeit/Boxenaufenthalt in Hochtief- als in } \\
\text { Hochboxen } \\
\text { Erhöhung der Liegedauer in weichen Sandboxen }\end{array}$ \\
\hline $\begin{array}{l}\text { Trockene - feuchte } \\
\text { Liegeflächen }\end{array}$ & $\begin{array}{l}(25) \\
(25)\end{array}$ & $\begin{array}{l}\text { Wahlversuch: Kühe wählten } 12,5 \text { Stunden/Tag trockene Liegeflä- } \\
\text { chen, 0,9 Stunden/Tag feuchte Liegeflächen } \\
\text { Verringerung der Liegezeit um } 5 \text { Stunden/Tag bei feuchtem Pols- } \\
\text { termaterial (26.5\% TS) verglichen mit trockenem Polstermaterial } \\
\text { (86.4\% TS). }\end{array}$ \\
\hline $\begin{array}{l}\text { Tag } 16 \text { ante partum - } \\
\text { Abkalbetag }\end{array}$ & $(12)$ & Reduktion der Liegezeit von 13,5 Stunden auf 10,8 Stunden \\
\hline Betrieb (Mittelwert) & (40) & $\begin{array}{l}\text { Liegedauer variierte zwischen } 9,5 \text { und } 12,9 \text { Stunden/Tag } \\
7 \text { bis } 10 \text { Liegephasen/Tag } \\
\text { Dauer einer Liegephase } 65 \text { bis } 112 \text { Minuten }\end{array}$ \\
\hline Individuum & $\begin{array}{l}(23) \\
(40)\end{array}$ & $\begin{array}{l}\text { Liegedauer variierte von } 5,6 \text { bis } 17,5 \text { Stunden/Tag } \\
\text { Liegedauer variierte von } 4,3 \text { bis } 19,5 \text { Stunden/Tag }\end{array}$ \\
\hline Tier-Boxen-Verhältnis & $(24)$ & $\begin{array}{l}\text { Reduktion der Liegezeiten um 1,7 Stunden von einem Verhältnis } \\
\text { von } 12 \text { Tieren zu } 12 \text { Boxen bis zu einem Verhältnis von } 12 \text { Tieren } \\
\text { zu } 8 \text { Boxen }\end{array}$ \\
\hline $\begin{array}{l}\text { Alter Stall/Stallneubau } \\
\text { mit Vergrösserung der } \\
\text { Liegefläche }\end{array}$ & $(53)$ & $\begin{array}{l}\text { Verlängerung der Liegedauer von } 9 \mathrm{~h} 26 \mathrm{~min} \text { auf } 11 \mathrm{~h} 45 \mathrm{~min} . \\
\text { Verminderung der Liegeperioden von } 12,3 \text { auf } 10,5 \\
73,1 \% \text { ungehindertes Abliegen im alten und } 85,9 \% \text { im neuen Stall } \\
\text { signifikante Abnahme von Integumentschäden und Umfangsver- } \\
\text { mehrungen im neuen Stall }\end{array}$ \\
\hline Lahmheit & (8) & Lahme Tiere liegen 13 Stunden, nicht lahme 10,9 Stunden/Tag \\
\hline
\end{tabular}


Tab. 3 Angaben zu Abmessungen von Liegeboxen (in Zentimetern) in der Schweiz*(94), Deutschland $* *(42,68)$ und USA $* * *(16)$

\begin{tabular}{|c|c|c|c|c|c|c|}
\hline \multirow[b]{2}{*}{ Boxenmaß (cm) } & \multicolumn{3}{|c|}{$\begin{array}{c}\text { Kühe und hochträch- } \\
\text { tige Erstkalbende* } \\
\text { mit Widerristhöhe } \\
\text { von cm }\end{array}$} & \multirow[t]{2}{*}{$\begin{array}{l}\text { Kühe**, } \\
\text { nicht näher } \\
\text { bezeichnet }\end{array}$} & \multirow{2}{*}{$\begin{array}{c}\begin{array}{c}\text { Erst- } \\
\text { laktierende*** }\end{array} \\
636 \mathrm{~kg} \text { Le- } \\
\text { bendmasse }\end{array}$} & \multirow{2}{*}{$\begin{array}{c}\begin{array}{c}\text { Ausgewachsene } \\
\text { Kuh*** }\end{array} \\
\begin{array}{c}727 \text { kg Lebend- } \\
\text { masse }\end{array}\end{array}$} \\
\hline & $\begin{array}{c}125 \\
5\end{array}$ & $\begin{array}{c}135 \pm \\
5\end{array}$ & $\begin{array}{c}145 \pm \\
5\end{array}$ & & & \\
\hline $\begin{array}{l}\text { Boxenlänge wand- } \\
\text { ständig }\end{array}$ & 230 & 240 & 260 & 270 & 274 & 305 \\
\hline $\begin{array}{l}\text { Boxenlänge gegen- } \\
\text { ständig }\end{array}$ & 200 & 220 & 235 & 240 & 259 & 274 \\
\hline Liegelänge & 165 & 185 & 190 & $173-178$ & $173-178$ & $178-183$ \\
\hline $\begin{array}{l}\text { Kopfraum bei wand- } \\
\text { ständiger Boxe }\end{array}$ & 65 & 55 & 70 & $97-92$ & $96-101$ & $122-127$ \\
\hline $\begin{array}{l}\text { Horizontale Entfer- } \\
\text { nung Nackenrohr- } \\
\text { Boxenkante }\end{array}$ & & & & $170-175$ & $173-178$ & $178-183$ \\
\hline $\begin{array}{l}\text { Diagonale Boxen- } \\
\text { kante-Nackenrohr }\end{array}$ & & & & 2,15 & & \\
\hline Boxenbreite & 110 & 120 & 125 & 122 & 122 & 127 \\
\hline Höhe des Bugbretts & & & & $10-20$ & 10 & 10 \\
\hline $\begin{array}{l}\text { Höhe der unteren } \\
\text { Seitenbegrenzung }\end{array}$ & 40 & 40 & 40 & $25-30$ & 30 & 30 \\
\hline $\begin{array}{l}\text { Höhe des Nacken- } \\
\text { rohrs }\end{array}$ & & & & $120-125$ & 122 & 127 \\
\hline Höhe Boxenkante & & & & $20-30$ & 20 & 20 \\
\hline $\begin{array}{l}\text { Gefälle der Liegeflä- } \\
\text { che (Hochboxe) }\end{array}$ & & $-5 \%(9$ & & $\begin{array}{c}2-4 \%(41 \\
68)\end{array}$ & & \\
\hline
\end{tabular}




\section{Abbildungslegenden}

Abb. 1 Schädigungen an der lateralen Seite des Tarsus im Röntgen- und Ultraschallbild a) Brown-Swiss-Kuh, 4 Jahre, Röntgenaufnahme des rechten Tarsus im plantaro-dorsalen Strahlengang. Chronisches Hygrom mit größter Ausdehnung über dem Os malleolare b) Schweizer Original Braunviehkuh, 3 Jahre, Ultraschallbild. Entzündliches Ödem mit Auflockerung der Subkutis und der periartikulären Weichteile (kurze Pfeile).

Fig. 1: Lesions at the lateral aspect of the hock in a radiograph and an ultrasonographic picture

a) Brown Swiss Cow, 4 years, radiograph, plantaro-dorsal view. Chronic hygroma centred over the malleolar bone.

b) Original Brown Swiss Cow, 3 years, Ultrasonographic picture. Inflammatory oedema, disruption of the subcutis and fluid accumulation in the periarticular tissue (short arrows).

Abb. 2 Diagramm zur „Kartographierung“ von Schädigungen der Haut und von tieferen Geweben am Tarsus des Rindes (nach (74)). Haarverlust, Dekubitus und Umfangsvermehrungen werden jeweils getrennt bewertet.

Fig. 2: Diagram illustrating the mapping of lesions (74) of the skin and underlying tissues of the hock in cattle. Hair loss, severity of decubitus ulcers and swelling are graded on separate diagrams.

Abb. 3 Beispiele für Haarverlust und Dekubitalschädigungen an der Lateralseite des Tarsus. Von links nach rechts: Beispiele für Grad 1 - Hautrötung und leichte Umfangsvermehrung, Grad 2 = Durchbrechen der Hautbarriere und stärkere Umfangsvermehrung, Grad 3 = Großflächige Hautrötung mit zentraler Ulzeration, starke Umfangsvermehrung.

Fig. 3: Examples for hair loss and decubitus ulcers on the lateral aspect of the hock. Examples for Grade 1 -3, from left to right; a) grade 1, Hair loss, erythema and swelling; b) grade 2, broken skin and swelling; c) grade 3, Considerable swelling with hair loss and central decubitus ulcer. 
Abb. 4 Anbindestall, kurze Liegefläche mit starkem Gefälle (7 \%), schadhaften Gummimatten und Gitterrost. Die Kühe liegen mit den Sprunggelenken auf dem Gitterrost. Bestandproblem: Sprunggelenkschädigungen und Sohlengeschwüre.

Fig. 4: Tie stall with short lying surface and $7 \%$ slope, old rubber mats and gutter grates. The cows lie with their hocks on the grates. Herd problems were tarsal swellings/lesions and sole ulcers.

Abb. 5 a und b Beispiele für unzureichende Kompensationsreaktionen des Körpers auf die Druckeinwirkung an der Lateralseite des Tarsus. a) Dekubitalnekrose an der Außenseite des Sprunggelenks mit Einbruch in das Tarsokruralgelenk, Schlachtung; b) sehr großes Hygrom, Exstirpation in toto unter Sekundärheilung der Haut.

Fig. 5 a and b: Examples of abnormal reaction to chronic pressure on the lateral aspect of the hock in cattle. a) Necrotising decubitus ulcer on the lateral aspect of the hock involving the tarsiocrural joint, which necessitated slaughter of the patient. b) Very large hygroma on the lateral tarsal area; resolved via surgical removal and healing of the skin by second intention. 


\section{Literaturverzeichnis}

1. Scientific Opinion of the Panel on Animal Health and Welfare on a request from European Commission on the overall effects of farming systems on dairy cow welfare and disease. EFSA $J$ 2009; 1143: 1-38.

2. Alban L, Agger JF, Lawson LG. Lameness in tied Danish dairy cattle: The possible influence of housing systems, management, milk yield, and prior incidents of lameness. Prev Vet Med 1996; 29: $135-49$.

3. Andreasen SN, Forkman B. The welfare of dairy cows is improved in relation to cleanliness and integument alterations on the hocks and lameness when sand is used as stall surface. J Dairy Sci 2012; 95: 4961-7.

4. Bareille N, Beaudeau F, Billon S, Robert A, Faverdin P. Effects of health disorders on feed intake and milk production in dairy cows. Livest Prod Sci 2003; 83: 53-62.

5. Barker ZE, Amory JR, Wright JL, Mason SA, Blowey RW, Green LE. Risk factors for increased rates of sole ulcers, white line disease, and digital dermatitis in dairy cattle from twenty-seven farms in England and Wales. J Dairy Sci 2009; 92: 1971-8.

6. Barker ZE, Leach KA, Whay HR, Bell NJ, Main DC. Assessment of lameness prevalence and associated risk factors in dairy herds in England and Wales. J Dairy Sci 2010; 93: 932-41.

7. Bernardi F, Fregonesi J, Winckler C, Veira DM, von Keyserlingk MA, Weary DM. The stalldesign paradox: neck rails increase lameness but improve udder and stall hygiene. J Dairy Sci 2009; 92: 3074-80.

8. Blackie N, Bleach E, Amorya J, Scaife J. Impact of lameness on gait characteristics and lying behaviour of zero grazed dairy cattle in early lactation. Appl Anim Behav Sci 2011; 129: 67-73.

9. Bünger A. Die Länge des produktiven Lebens und ihre Beziehung zu linearen Exterieurmerkmalen bei Holstein-Friesian-Kühen. Inaugural-Dissertation Universität Göttingen 1999.

10. Busato A, Trachsel P, Blum JW. Frequency of traumatic cow injuries in relation to housing systems in Swiss organic dairy herds. J Vet Med A Physiol Pathol Clin Med 2000; 47: 221-9.

11. Calamari L, Calegari F, Stefanini L. Effect of different free stall surfaces on behavioural, productive and metabolic parameters in dairy cows. Appl Anim Behav Sci 2009; 120: 9-17.

12. Calderon DF, Cook NB. The effect of lameness on the resting behavior and metabolic status of dairy cattle during the transition period in a freestall-housed dairy herd. J Dairy Sci 2011; 94: 2883-94.

13. Camiloti TV, Fregonesi JA, von Keyserlingk MA, Weary DM. Short communication: effects of bedding quality on the lying behavior of dairy calves. J Dairy Sci 2012; 95: 3380-3.

14. Chapinal N, de Passille AM, Rushen J, Wagner S. Automated methods for detecting lameness and measuring analgesia in dairy cattle. J Dairy Sci 2010; 93: 2007-13.

15. Chaplin SJ, Tierney G, Stockwell C, Logue DN, Kelly M. An evaluation of mattresses and mats in two dairy units. Appl Anim Behav Sci 2000; 66: 263-72.

16. Cook NB, Nordlund KV. Behavioral needs of the transition cow and considerations for special needs facility design. Vet Clin North Am Food Anim Pract 2004; 20: 495-520.

17. Cramer G, Lissemore KD, Guard CL, Leslie KE, Kelton DF. Herd-level risk factors for seven different foot lesions in Ontario Holstein cattle housed in tie stalls or free stalls. J Dairy Sci 2009; 92: 1404-11. 
18. Dippel S, Dolezal M, Brenninkmeyer C, Brinkmann J, March S, Knierim U, Winckler C. Risk factors for lameness in cubicle housed Austrian Simmental dairy cows. Prev Vet Med 2009; 90: 10212.

19. Drissler M, Gaworski M, Tucker CB, Weary DM. Freestall maintenance: effects on lying behavior of dairy cattle. J Dairy Sci 2005; 88: 2381-7.

20. Eilers U. Liegeboxenmasse: Kompromissformel für Komfort. Bericht. Aulendorf: Bildungsund Wissenszentrum Baden-Württemberg 2007; 1-12.

21. Ekesbo I. Disease incidence in tied and loose housed dairy cattle. PhD-Arbeit. Stockholm: Royal Veterinary College; 1966.

22. Falk AC, Weary DM, Winckler C, von Keyserlingk MAG. Preference for pasture versus freestall housing by dairy cattle when stall availability indoors is reduced. J Dairy Sci 2012; 95: 6409-15.

23. Fregonesi JA, Leaver JD. Behaviour, performance and health indicators of welfare for dairy cows housed in strawyard or cubicle systems. Livest Prod Sci 2001; 68: 205-16.

24. Fregonesi JA, Tucker CB, Weary DM. Overstocking reduces lying time in dairy cows. J Dairy Sci 2007; 90: 3349-54.

25. Fregonesi JA, Veira DM, von Keyserlingk MA, Weary DM. Effects of bedding quality on lying behavior of dairy cows. J Dairy Sci 2007; 90: 5468-72.

26. Fregonesi JA, von Keyserlingk MA, Tucker CB, Veira DM, Weary DM. Neck-rail position in the free stall affects standing behavior and udder and stall cleanliness. J Dairy Sci 2009; 92: 1979-85.

27. Fulwider WK, Grandin T, Garrick DJ, Engle TE, Lamm WD, Dalsted NL, Rollin BE. Influence of free-stall base on tarsal joint lesions and hygiene in dairy cows. J Dairy Sci 2007; 90: 3559-66.

28. Fulwider WK, Grandin T, Rollin BE, Engle TE, Dalsted NL, Lamm WD. Survey of dairy management practices on one hundred thirteen north central and northeastern United States dairies. J Dairy Sci 2008; 91: 1686-92.

29. Haley DB, de Passille AM, Rushen J. Assessing cow comfort: effects of two floor types and two tie stall designs on the behaviour of lactating dairy cows. Appl Anim Behav Sci 2001; 71: 105-17.

30. Haskell MJ, Rennie LJ, Bowell VA, Bell MJ, Lawrence AB. Housing system, milk production, and zero-grazing effects on lameness and leg injury in dairy cows. J Dairy Sci; 89: 4259-66.

31. Hemsworth PH, Coleman GJ, Barnett JL, Borg S. Relationships between human-animal interactions and productivity of commercial dairy cows. J Anim Sci 2000; 78: 2821-31.

32. Hemsworth PH, Coleman GJ, Barnett JL, Borg S, Dowling S. The effects of cognitive behavioral intervention on the attitude and behavior of stockpersons and the behavior and productivity of commercial dairy cows. J Anim Sci 2002; 80: 68-78.

33. Hernandez-Mendo O, von Keyserlingk MA, Veira DM, Weary DM. Effects of pasture on lameness in dairy cows. J Dairy Sci 2007; 90: 1209-14.

34. Hernandez J, Shearer JK, Webb DW. Effect of lameness on the calving-to-conception interval in dairy cows. J Am Vet Med Assoc 2001; 218: 1611-4.

35. Hoerning B, Hrsg. Nutzen und Kosten tiergerechter Milchviehhaltung - Versuch einer Bewertung verschiedener Laufstallsysteme anhand des Tierverhaltens und der Jahreskosten. Von LeitBildern zu Leit-Linien. Beitr 6 Wissenschaftstagung zum Ökologischen Landbau 2001; Dr. Köster, Berlin.

36. Huxley J, Whay H. Cow based assessments. Part 2: Rising restrictions and injuries associated with the lying surface. UK Vet 2006; 11: 1-6.

37. Huxley JN. Lameness in cattle: An ongoing concern. Vet J 2012; 193: 610-611. 
38. Huxley JN, Whay HR. Current attitudes of cattle practitioners to pain and the use of analgesics in cattle. Vet Rec 2006; 159: 662-8.

39. Ito K, von Keyserlingk MA, Leblanc SJ, Weary DM. Lying behavior as an indicator of lameness in dairy cows. J Dairy Sci 2010; 93: 3553-60.

40. Ito K, Weary DM, von Keyserlingk MA. Lying behavior: assessing within- and between-herd variation in free-stall-housed dairy cows. J Dairy Sci 2009; 92: 4412-20.

41. Jungbluth T, Wandel H. Was zeichnet eine tiergerechte Liegebox aus? Nutztierpraxis aktuell. 2004; 10: 45-7.

42. Kanswohl N, Sanftleben P. Analyse und Bewertung von Hoch- und Tiefboxen für Milchrinder aus arbeitswirtschaftlicher, ethologischer, hygienischer und ökonomischer Sicht. In: Sanftleben P, editor.: Landesforschungsanstalt für Landwirtschaft und Fischerei Mecklenburg-Vorpommern Institut für Tierproduktion 2006; 3-36.

43. Keil NM, Wiederkehr TU, Friedli K, Wechsler B. Effects of frequency and duration of outdoor exercise on the prevalence of hock lesions in tied Swiss dairy cows. Prev Vet Med 2006; 74: 14253.

44. Kielland C, Boe KE, Zanella AJ, Osteras O. Risk factors for skin lesions on the necks of Norwegian dairy cows. J Dairy Sci 2010; 93: 3979-89.

45. Kielland C, Ruud LE, Zanella AJ, Osteras O. Prevalence and risk factors for skin lesions on legs of dairy cattle housed in freestalls in Norway. J Dairy Sci 2009; 92: 5487-96.

46. Klaas IC, Rousing T, Fossing C, Hindhede J, Sorensen JT. Is lameness a welfare problem in dairy farms with automatic milking systems? Anim Welf 2003; 12: 599-603.

47. Klee W, Hänichen T. Epidemiologische, klinische und pathologisch-anatomische Untersuchung über die Entzündung der Karpalgelenkstrecker beim Rind. Schweiz Arch Tierheilk 1989; 131: $151-7$.

48. Knell H-G. Umbau von Hochboxen zu Hochtiefboxen - Auswirkungen auf Verhalten, Gliedmassengesundheit und Milchleistung, Masterarbeit. Justus-Liebig-Universität Giessen 2008.

49. Knierim U, Winckler C. On-farm welfare assessment in cattle: validity, reliability and feasibility issues and future perspectives with special regard to the Welfare Quality (R) approach. Anim Welfare 2009; 18: 451-8.

50. Krohn CC, Munksgaard L. Behavior of Dairy-Cows Kept in Extensive (Loose Housing Pasture) or Intensive (Tie Stall) Environments .2. Lying and Lying-down Behavior. Appl Anim Behav Sci 1993; 37: 1-16.

51. Kümper H. Entstehungsweise, Therapie und Prophylaxe von Gliedmassenerkrankungen bei Kühen. Grosstierpraxis 2000; 1: 6-24.

52. Laven R, Livesey C. Getting to grips with hock lesions in cattle. Vet Rec 2011 10; 169: 632-3.

53. Liebhart S. Auswirkungen einer Änderung der Haltungsumwelt auf ethologische, morphologische und hygienische Parameter einer Milchviehherde, Inaugural-Dissertation. Ludwig-MaximiliansUniversität München 2009.

54. Lievaart JJ, Noordhuizen JP. Ranking experts' preferences regarding measures and methods of assessment of welfare in dairy herds using Adaptive Conjoint Analysis. J Dairy Sci 2011; 94: 3420-7.

55. Livesey CT, Marsh C, Metcalf JA, Laven RA. Hock injuries in cattle kept in straw yards or cubicles with rubber mats or mattresses. Vet Rec 2002; 150: 677-9.

56. Lobeck KM, Endres MI, Shane EM, Godden SM, Fetrow J. Animal welfare in crossventilated, compost-bedded pack, and naturally ventilated dairy barns in the upper Midwest. J Dairy Sci 2011; 94: 5469-79. 
57. Lombard JE, Tucker CB, von Keyserlingk MAG, Kopral CA, Weary DM. Associations between cow hygiene, hock injuries, and free stall usage on US dairy farms. J Dairy Sci 2010; 93: 466876.

58. Manninen E, de Passille AM, Rushen J, Norring M, Saloniemi H. Preferences of dairy cows kept in unheated buildings for different kind of cubicle flooring. Appl Anim Behav Sci 2002; 75: 28192.

59. McConnel CS, Lombard JE, Wagner BA, Garry FB. Evaluation of factors associated with increased dairy cow mortality on United States dairy operations. J Dairy Sci 2008; 91: 1423-32.

60. Melendez P, Bartolome J, Archbald LF, Donovan A. The association between lameness, ovarian cysts and fertility in lactating dairy cows. Theriogenology 2003; 59: 927-37.

61. Mulleder C, Troxler J, Laaha G, Waiblinger S. Can environmental variables replace some animal-based parameters in welfare assessment of dairy cows? Anim Welf 2007; 16: 153-6.

62. Müller M. Dekubitus beim Rind. Grosstierpraxis. 2004; 5: 22-8.

63. Munksgaard L, Jensen MB, Pedersen LJ, Hansen SW, Matthews L. Quantifying behavioural priorities-effects of time constraints on behaviour of dairy cows, Bos taurus. Appl Anim Behav Sci 2005; 92: 3-14.

64. N.N. The Effect of Neck Rail and Mounting Rail Position on Cow Behavior. In: http://www.omafra.gov.on.ca/ english/livestock/dairy/facts.

65. Norring M, Manninen E, de Passille AM, Rushen J, Munksgaard L, Saloniemi H. Effects of sand and straw bedding on the lying behavior, cleanliness, and hoof and hock injuries of dairy cows. $\mathrm{J}$ Dairy Sci 2008; 91: 570-6.

66. Norring M, Manninen E, de Passille AM, Rushen J, Saloniemi H. Preferences of dairy cows for three stall surface materials with small amounts of bedding. J Dairy Sci 2010; 93: 70-4.

67. Nuss K, Räber M, Sydler T, Muggli E, Hässig M, Guscetti F. Bursitis mit schwerwiegenden Sehnen- und Muskelnekrosen an der Aussenseite der Kniegelenke bei 21 Rindern. Schweiz Arch Tierheilk 2011; 153: 520-5.

68. Pelzer A. Planungshinweise zur Liegeboxengestaltung für Milchkühe, Merkblatt 341. Frankfurt/Main, DLG-Verlag; 2007.

69. Piguet M, Steiner A, Eicher R, Martig J. [Surgical treatment of carpal hygroma in cattle: 17 cases (1990-1994)]. Schweiz Arch Tierheilkd 1997; 139: 210-6.

70. Plesch G, Broerkens N, Laister S, Winckler C, Knierim U. Reliability and feasibility of selected measures concerning resting behaviour for the on-farm welfare assessment in dairy cows. Appl Anim Behav Sci 2010; 126: 19-26.

71. Potterton SL, Green MJ, Harris J, Millar KM, Whay HR, Huxley JN. Risk factors associated with hair loss, ulceration, and swelling at the hock in freestall-housed UK dairy herds. J Dairy Sci 2011; 94: 2952-63.

72. Potterton SL, Green MJ, Millar KM, Brignell CJ, Harris J, Whay HR, Huxley JN. Prevalence and characterisation of, and producers' attitudes towards, hock lesions in UK dairy cattle. Vet Rec 2011; 169: 634-47.

73. Regula G, Danuser J, Spycher B, Wechsler B. Health and welfare of dairy cows in different husbandry systems in Switzerland. Prev Vet Med 2004; 66: 247-64.

74. Reich LJ, Weary DM, Veira DM, von Keyserlingk MA. Effects of sawdust bedding dry matter on lying behavior of dairy cows: a dose-dependent response. J Dairy Sci 2010; 93: 1561-5. 
75. Rouha-Mulleder C, Iben C, Wagner E, Laaha G, Troxler J, Waiblinger S. Relative importance of factors influencing the prevalence of lameness in Austrian cubicle loose-housed dairy cows. Prev Vet Med 2009; 92: 123-33.

76. Rutherford KM, Langford FM, Jack MC, Sherwood L, Lawrence AB, Haskell MJ. Hock injury prevalence and associated risk factors on organic and nonorganic dairy farms in the United Kingdom. J Dairy Sci 2008; 91: 2265-74.

77. Sogstad AM, Osteras O, Fjeldaas T. Bovine claw and limb disorders related to reproductive performance and production diseases. J Dairy Sci 2006; 89: 2519-28.

78. Spycher B, Regula G, Wechsler B, Danuser J. Gesundheit und Wohlergehen von Milchkuhen in verschiedenen Haltungsprogrammen. Schweiz Arch Tierheilk 2002; 144: 519-30.

79. Stöber M. Einführung. In: Innere Medizin und Chirurgie des Rindes, 4 Aufl. Dirksen G, Gründer H-D, Dirksen G, Hrsg. Berlin, Wien: Parey 2002; 1-21.

80. Strebel M. Zu den Sprunggelenkkrankheiten beim Rinde. Schweiz Arch Tierheilk 1895; 37: 82-90.

81. Telezhenko E, von Keyserlingk MA, Talebi A, Weary DM. Effect of pen size, group size, and stocking density on activity in freestall-housed dairy cows. J Dairy Sci 2012; 95: 3064-9.

82. Thomsen PT, Dahl-Pedersen K, Jensen HE. Necropsy as a means to gain additional information about causes of dairy cow deaths. J Dairy Sci 2012; 95, 5798-5803.

83. Tucker CB, Weary DM, Fraser D. Effects of three types of free-stall surfaces on preferences and stall usage by dairy cows. J Dairy Sci 2003; 86: 521-9.

84. Tucker CB, Weary DM, Fraser D. Free-stall dimensions: effects on preference and stall usage. J Dairy Sci 2004; 87: 1208-16.

85. Tucker CB, Weary DM, Fraser D. Influence of neck-rail placement on free-stall preference, use, and cleanliness. J Dairy Sci 2005; 88: 2730-7.

86. Tucker CB, Weary DM, von Keyserlingk MA, Beauchemin KA. Cow comfort in tie-stalls: increased depth of shavings or straw bedding increases lying time. J Dairy Sci 2009; 92: 2684-90.

87. van Gastelen S, Westerlaan B, Houwers DJ, van Eerdenburg FJ. A study on cow comfort and risk for lameness and mastitis in relation to different types of bedding materials. . J Dairy Sci 2011; 94: 4878-88.

88. Wassmuth R. Ansprüche der Milchkühe an das Haltungssystem. In: Landtechnisch-Bauliche Jahrestagung. Wendl G, Hrsg. Marktoberdorf: Tagungsband 2001; 23-30.

89. Weary DM, Taszkun I. Hock lesions and free-stall design. J Dairy Sci 2000; 83: 697-702.

90. Weary DM, Tucker CB. The science of cow comfort. Proceedings of the Joint Meeting of the Ontario Agri Business Association and the Ontario Association of Bovine Practitioners. Guelph, Ontario, Canada2003; $1-15$.

91. Whay HR, Main DC, Green LE, Webster AJ. Assessment of the welfare of dairy cattle using animal-based measurements: direct observations and investigation of farm records. Vet Rec 2003; 153: 197-202.

92. Willen S. Tierbezogene Indikatoren zur Beurteilung der Tiergerechtheit in der Milchviehhaltung - methodische Untersuchungen und Beziehungen zum Haltungssystem. Inaugural-Dissertation, Tierärztliche Hochschule Hannover; 2004.

93. Zaffino J. An evaluation of hock, knee, and neck injuries. Master of Science Thesis, University of Guelph; 2012.

94. Zähner M. ART-Baumerkblätter. In: ART FAR-T, editor. Tänikon: Eigenössisches Volkswirtschaftsdepartement EVD; 2009. p. 1-7. 
95. Zerzawy B. Haltungsbedingte, adspektorisch und palpatorisch erfaßbare Krankheiten und Abgangsursachen von Milchkühen in Abhängigkeit von den Stallverhältnissen im Liegeboxenlaufstall. Inaugural-Dissertation, Justus-Liebig-Universität Giessen; 1989.

96. Zurbrigg K, Kelton D, Anderson N, Millman S. Stall dimensions and the prevalence of lameness, injury, and cleanliness on 317 tie-stall dairy farms in Ontario. Can Vet J 2005; 46: 902-9.

97. Zurbrigg K, Kelton D, Anderson N, Millman S. Tie-stall design and its relationship to lameness, injury, and cleanliness on 317 Ontario dairy farms. J Dairy Sci 2005; 88: 3201-10. 